\title{
Trapped Fermi Gases in Rotating Optical Lattices: Realization and Detection of the Topological Hofstadter Insulator
}

\author{
R. O. Umucalılar, ${ }^{1}$ Hui Zhai, ${ }^{2,3}$ and M. Ö. Oktel ${ }^{1, *}$ \\ ${ }^{1}$ Department of Physics, Bilkent University, 06800 Ankara, Turkey \\ ${ }^{2}$ Department of Physics, University of California, Berkeley, California, 94720, USA \\ ${ }^{3}$ Materials Sciences Division, Lawrence Berkeley National Laboratory, Berkeley, California, 94720, USA \\ (Received 23 November 2007; revised manuscript received 9 January 2008; published 19 February 2008) \\ We consider a gas of noninteracting spinless fermions in a rotating optical lattice and calculate the \\ density profile of the gas in an external confinement potential. The density profile exhibits distinct \\ plateaus, which correspond to gaps in the single particle spectrum known as the Hofstadter butterfly. The \\ plateaus result from insulating behavior whenever the Fermi energy lies within a gap. We discuss the \\ necessary conditions to realize the Hofstadter insulator in a cold atom setup and show how the quantized \\ Hall conductance can be measured from density profiles using the Středa formula.
}

Recently, some fundamental models of many-particle quantum systems have been experimentally realized using trapped ultracold fermions. Some of these experiments, such as transport in optical lattices [1], have demonstrated well-known effects with improved precision, while others, such as spin imbalanced superfluidity [2,3], have provided access to previously unexplored regimes. With constantly improving experimental control over ultracold systems, it is expected that many other fundamental ideas can be tested in the laboratory for the first time.

A basic problem in quantum mechanics is the dynamics of a charged particle moving in a periodic potential under a magnetic field. The single particle spectrum depends sensitively on the ratio of the flux through a unit cell of the lattice to flux quantum. For a tight-binding lattice, a single band splits into narrow magnetic bands, forming a selfsimilar energy spectrum known as the Hofstadter butterfly [4]. The gaps in the Hofstadter spectrum form continuous regions for a finite range of flux. For a system of noninteracting fermions, it was shown by Thouless et al. that whenever the Fermi energy lies in one of these gaps, the Hall conductance of the system is quantized [5]. This quantization is topological in nature, and the quantized Hall conductance is determined uniquely by the magnetic translation symmetry [6]. This Hofstadter insulating phase is a topological insulator that is characterized by two topological numbers [7], the first Chern number corresponding to Hall conductance and another number related to polarizability [8].

Despite its mathematical elegance, the Hofstadter insulator can hardly be achieved in solid state systems because the magnetic field needs to be thousands of Tesla in order to create a magnetic flux which can be comparable to one flux quantum per unit cell [9]. While in some experiments superlattice structures have been used to study the splitting of Landau levels under a periodic potential, the tightbinding regime has never been experimentally realized [10]. The main purpose of this Letter is to propose an alternative way to achieve and to experimentally study this topological insulator by using ultracold Fermi gases in a rotating optical lattice. We discuss (i) the conditions to realize the Hofstadter insulator in rotating optical lattices, and (ii) the manifestation of this insulator in real space density profile and the method to detect the Hall conductance in a cold atom setup.

The Hamiltonian for a particle in a rotating lattice is

$$
\begin{aligned}
H= & \frac{1}{2 m} \mathbf{p}_{\perp}^{2}+\frac{1}{2} m \omega_{\perp}^{2} r^{2}-\Omega \hat{\mathbf{z}} \cdot \mathbf{r} \\
& \times \mathbf{p}_{\perp}+V_{0}\left[\sin ^{2}(k x)+\sin ^{2}(k y)\right],
\end{aligned}
$$

where $m$ is the mass of the particle, $\Omega$ is the rotation frequency, and $\omega_{\perp}$ is the transverse trapping frequency. $\mathbf{p}_{\perp}=\left(p_{x}, p_{y}\right), \mathbf{r}=(x, y)$, and $V_{0}$ is the maximum depth of the optical potential created by a laser light with wave number $k=2 \pi / \lambda$ (for counterpropagating laser beams lattice constant $a$ is equal to $\lambda / 2$ ). Photon recoil energy $E_{R}$, defined as $\hbar^{2} k^{2} /(2 m)$ is introduced as the energy unit in the following discussion. This Hamiltonian can be rearranged as

$$
\begin{aligned}
H= & \frac{\left(\mathbf{p}_{\perp}-m \Omega \hat{\mathbf{z}} \times \mathbf{r}\right)^{2}}{2 m}+V_{0}\left[\sin ^{2}(k x)+\sin ^{2}(k y)\right] \\
& +\frac{1}{2} m\left(\omega_{\perp}^{2}-\Omega^{2}\right) r^{2} .
\end{aligned}
$$

When $\Omega$ is close to $\omega_{\perp}$, the third term represents a smooth potential in space. The first term describes the motion of a particle under a perpendicular magnetic field with strength $B=2 m c \Omega / e$. This effective magnetic field description has led a number of authors to study the quantum-Hall type of physics in rotating quantum gases [11]. Here, we consider the presence of both rotation and a lattice potential [12], employing the Hofstadter model, which is appropriate for the description of a noninteracting gas of fermions in a lattice. 
We now discuss the conditions for simulating the Hofstadter model in an ultracold optical lattice system.

(1) The Hofstadter model is expressly single band; the motion of fermions in the periodic potential has to be well described by a single-band tight-binding model with nearest neighbor hopping. This requirement means that there has to be a finite gap between $s$ and $p$ bands, and the dispersion of the $s$ band can be well approximated by a cosine function. From the band-structure calculations for optical lattice potentials, one can easily show that this requirement is fulfilled when $V_{0}>3 E_{R}$ [13]. A rotating lattice experiment has recently been carried out [14]. Although in this particular experiment $V_{0}<1 E_{R}$, which is not deep enough to reach the tight-binding regime, there is no fundamental reason against increasing $V_{0}$ a few more $E_{R}$, as it has been routinely done in static lattice experiments [15].

(2) The "magnetic field" has to be strong enough. The dimensionless parameter $\phi=a^{2} B /(h c / e)$ is the magnetic flux quantum per plaquette and is connected to the rotation frequency $\Omega$ as $\phi=2 m a^{2} \Omega / h$. For rotation to create an effective magnetic flux close to 1 flux quantum, the rotation frequency $\Omega$ must be close to $E_{R} / \hbar$ of the lattice. In typical optical lattice experiments the recoil frequency is a few $\mathrm{kHz}$; thus, rotation of the lattice at hundreds to thousands of $\mathrm{Hz}$ would be enough to reach the high magnetic field limit.

(3) To observe the insulating behavior, the temperature has to be lower than the gap of the insulator. The gap for the Hofstadter insulator is comparable to the hopping amplitude $t$. In a moderately deep lattice, for which $V_{0} \sim 3-7 E_{R}$, for instance, $t$ is of the order of $1-10 \mathrm{nK}$. This is below currently attainable temperatures. However, cooling fermions to this regime is not more difficult than achieving degenerate Fermi gases in a lattice, and the latter is now the major goal pursued in many key laboratories in this field.

(4) The Hofstadter model is a noninteracting one. Strong repulsive or attractive interactions can lead to either exciton instability or BCS instability of the insulating phase (which will be discussed elsewhere), and therefore diminish its topological behavior. In ultracold atom experiments a single species of fermions is naturally noninteracting due to the Pauli exclusion principle.

Even when the above conditions are satisfied, there are two factors which complicate the correspondence between the results of ultracold atom experiments and theories developed for "bulk" systems. The first is the presence of an external confining potential in all ultracold atom experiments, i.e., the third term in Eq. (2). The second factor is that transport measurements are usually very hard for cold atom systems. Hereafter, we first calculate the density profile of a noninteracting Fermi gas in a rotating optical lattice, in the presence of a smooth external potential [16]. We find that the presence of the residual trapping potential does not preclude the observation of the effects of single particle spectrum, as long as it is varying smooth enough on the lattice length scale. Secondly, we show that one of the most important transport properties, namely, the Hall conductance which reflects the topological nature, can be inferred from the measurement of the density profile due to the well-known Středa formula [17].

When the residual trapping potential is slowly varying, we can utilize the local-density approximation (LDA) in which we define a local chemical potential $\mu_{l}(r)$ (or Fermi energy) as

$$
\mu_{l}(\mathbf{r})=\mu-V(\mathbf{r})
$$

where in our case $V(r)=m\left(\omega_{\perp}^{2}-\Omega^{2}\right) r^{2} / 2$. In what follows, we shall denote $\left(\omega_{\perp}^{2}-\Omega^{2}\right)$ by $\omega^{2}$.

To find the density profile $n(r)$, we simply count the number of states below $\mu_{l}(r)$ for the corresponding uniform system as

$$
n(r)=\int d \epsilon D(\epsilon) \Theta\left(\mu_{l}(r)-\epsilon\right) .
$$

So, we shall first find the density of states $D(\epsilon)$ for noninteracting fermions in a periodic lattice under a magnetic field. By using the Landau gauge $\vec{A}=B x \hat{y}$, one can construct the Hamiltonian for this system as follows:

$$
H=-t \sum_{\langle i, j\rangle} a_{i}^{\dagger} a_{j} e^{i A_{i j}},
$$

where $a_{i}\left(a_{i}^{\dagger}\right)$ is the fermionic annihilation (creation) operator at site $i$ and the sum is over nearest neighbor sites. Magnetic field affects the Hamiltonian through $A_{i j}$, which is equal to $\pm 2 \pi n \phi$, if $i$ and $j$ have the same $x$ coordinate $n a$, and is 0 otherwise, with the sign being determined by the hopping direction. The hopping strength $t$ can be obtained as a function of $V_{0} / E_{R}$ from a simple bandstructure calculation. The density of states is displayed in Fig. 1. When $\phi$ is a rational number $p / q$, with $p$ and $q$ being relatively prime integers, the energy band divides into $q$ bands [4]. In this spectrum the gaps form continuous regions in $\phi-E$ plane, although the band edges are fractal. When $\mu$ lies in a gap, the system is an insulator, and as one changes $\phi$ and $\mu$, the topological nature and the Hall conductance of the insulator do not change as long as one remains within the same gap [5]. The largest two gaps correspond to insulators with Hall conductance $\sigma_{x y}= \pm 1$, and the second largest ones have Hall conductance $\sigma_{x y}=$ \pm 2 and so on, as marked in Fig. 1.

To calculate the integral in Eq. (4) efficiently, we note that if we take $q$ to be sufficiently large, the bandwidths of each subband become negligibly small. Counting the number of states then reduces to counting these bands in certain intervals. In our numerical procedure, we determine the subbands with their edge values $\varepsilon_{\text {edge }}$. So the number of states per plaquette in the two dimensional case can conveniently be expressed as 


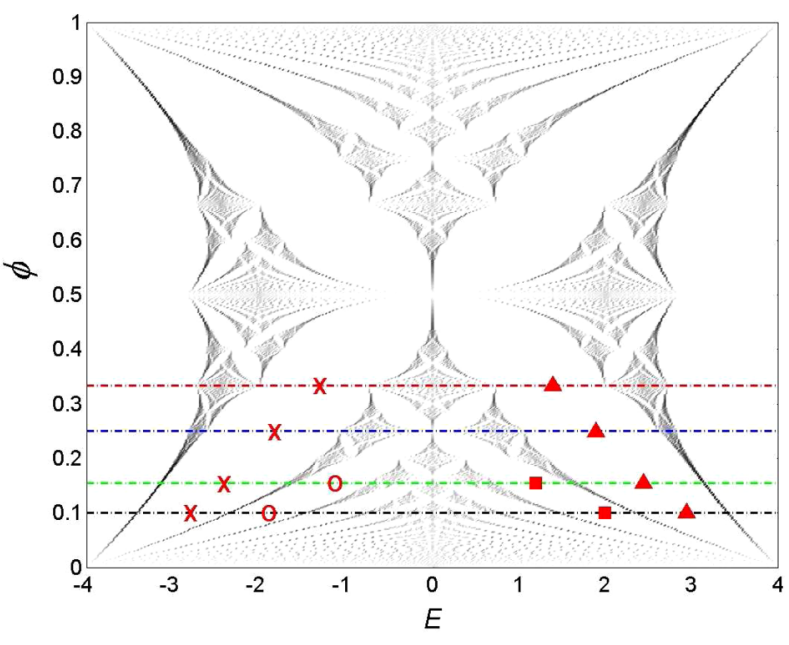

FIG. 1 (color online). Density of states for the Hofstadter butterfly. Darker regions imply greater density. Dashed lines represent the trajectory of local Fermi energy from the center to the edge of the cloud, for different values of $\phi$ corresponding to those used in Fig. 2, namely $\phi=1 / 3,1 / 4,1 / 7$, and 1/10. Regions marked by $\times$ and $\boldsymbol{\Delta}$ have Hall conductance $\sigma_{x y}= \pm 1$, and marked by $\bigcirc$ and $\mathbf{\square}$ have $\sigma_{x y}= \pm 2$.

$$
n(\mu)=\frac{1}{2 q} \sum_{\varepsilon_{\text {edge }}} \Theta\left(\mu-\varepsilon_{\text {edge }}\right) .
$$

In all of our calculations we took $q=401$, which is a prime number allowing $p$ to be successive integers. $\phi$ values for other small denominators of $q$ are approximated by properly choosing $p$. For instance, $\phi=1 / 10$ is approximated by $40 / 401,1 / 4$ by $100 / 401$, and $1 / 3$ by $134 / 401$.

We now present the density profiles for several $\phi$ values. To make a connection with experiments, we refer to the work in Ref. [18] in which ${ }^{40} \mathrm{~K}$ atoms are stored in an optical lattice with lattice constant $a=413 \mathrm{~nm}$. We take $V_{0}=5 E_{R}$, which gives $t=0.066 E_{R}$. The parameters at hand yield $E_{R} / \hbar=45.98 \mathrm{kHz}$ and $t / \hbar=3.035 \mathrm{kHz}$. With the choice $\omega \sim 355 \mathrm{~Hz}$, the gas extends over approximately 60 lattice sites in the radial direction, so that the assumption of LDA is satisfied. In Figs. 2 and 3 we fixed the number of fermions at 5000 .

When the local chemical potential $\mu_{l}(r)$ lies in one of the gaps, we have $\partial n(r) / \partial \mu(r)=0$ because of vanishing compressibility. Hence, as one can see by comparing Figs. 1 and 2, corresponding to the energy gaps in the single particle spectrum, there appear plateaus in the density profile. The discernible number of plateaus is related to the size of the energy gaps. For instance, in Fig. 2(a), the plateau with $n=1$ is the band insulator with completely filled band, which is topologically trivial and has vanishing Hall conductance. Apart from that, for $\phi=1 / 3$, the chemical potential trajectory passes through two gap regions which gives two plateaus with $n=0.333$ and $n=$ 0.667 , respectively. While for $\phi=1 / 4$, there are totally four subbands, but two of them touch at $\mu=0$, so there are
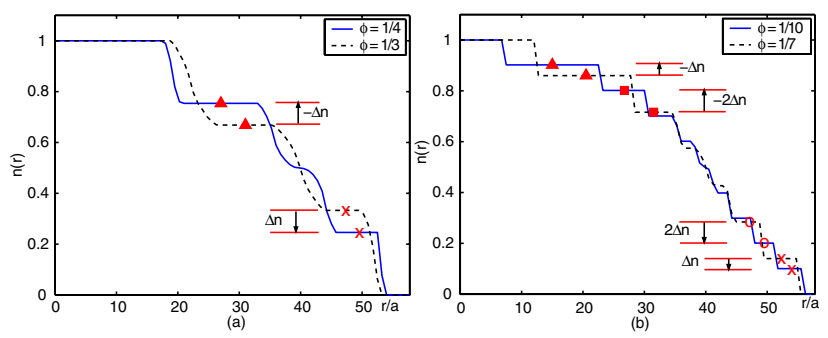

FIG. 2 (color online). (a) Density profiles for 5000 fermions with $\phi=1 / 4, \Omega=7.2992 \mathrm{kHz}, \omega_{\perp}=7.3078 \mathrm{kHz}$ (solid line) and $\phi=1 / 3, \Omega=9.7809 \mathrm{kHz}, \omega_{\perp}=9.7873 \mathrm{kHz}$ (dashed line). (b) Density profiles for 5000 fermions with $\phi=1 / 10$, $\Omega=2.9197 \mathrm{kHz}, \omega_{\perp}=2.9412 \mathrm{kHz}$ (solid line) and $\phi=1 / 7$, $\Omega=4.1605 \mathrm{kHz}, \omega_{\perp}=4.1756 \mathrm{kHz}$ (dashed line). Length is measured in units of lattice constant $a$. Density is given in units of particles per lattice site.

also two gap regions corresponding to two plateaus with $n=0.25$ and $n=0.75$. In Fig. 2(b) we choose two $\phi$ 's with larger $q$, where there are more gaps in the spectrum and therefore more density plateaus. Experimentally, the smaller gap one wants to find, the more difficult it is, because it requires larger system size and lower temperature.

In Fig. 3 we show the temperature effect on the visibility of plateaus. We implement the effect of finite temperature by incorporating the Fermi-Dirac distribution into our calculations as

$$
n_{2 D}\left(\mu_{l}(r), T\right)=\frac{1}{2 q} \sum_{\varepsilon_{\text {edge }}} \frac{1}{\exp \left[\left(\varepsilon_{\text {edge }}-\mu_{l}(r)\right) / k_{B} T\right]+1} .
$$

We observe from Fig. 3 that plateaus will be smeared out when $k_{B} T>0.5 t$.

As shown by Thouless et al., the topological distinction of the insulators we consider manifests itself in the Hall conductance, which should be quantized in units of $e^{2} / h$ [5]. Here, we propose a method to read out the information

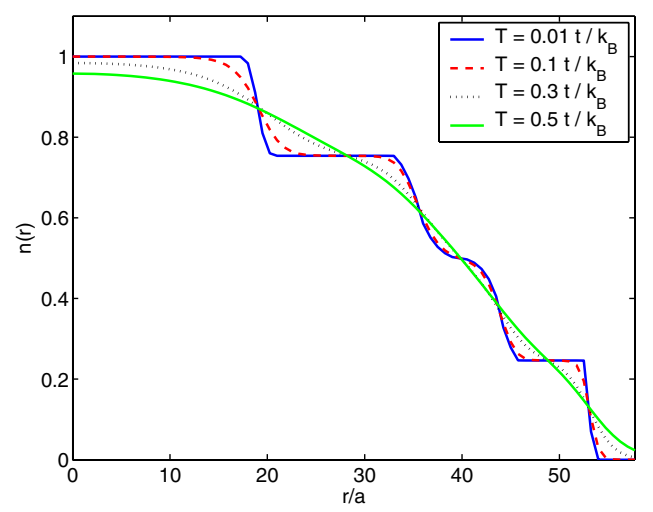

FIG. 3 (color online). Density profile for 5000 fermions at several temperatures when $\phi=1 / 4$. Plateaus become indiscernible when $k_{B} T \sim 0.5 t$. 
about the Hall conductance quantization from the obtained density profiles. One can observe how the particle density within a particular plateau (labeled by the corresponding gap) changes with the rotation frequency, and the change of plateau density with respect to rotation frequency is directly related to the Hall conductance of that plateau as follows.

Středa obtained a formula for the Hall conductance of a two dimensional charged system as

$$
\sigma_{x y}=e c \frac{\partial N}{\partial B},
$$

which is valid when the Fermi energy lies in a gap [17]. Here $N$ is the number of levels below the Fermi energy. For a neutral gas we can define a similar response function $\gamma_{x y}=J_{x} / F_{y}$, where $J_{x}$ is the mass current in $x$ direction induced by a force $F_{y}$ in the $y$ direction. Then the Streda formula for the rotating system can be written as

$$
\gamma_{x y}=\frac{1}{2} \frac{\partial N}{\partial \Omega} \text {. }
$$

Expressing the rotation frequency in terms of $\phi$ and $N$ in terms of the density per plaquette $n(r)$ (as in our density plots), one obtains

$$
\gamma_{x y}=\frac{m}{h} \frac{\partial n}{\partial \phi} .
$$

To measure Hall conductance, we first choose two $\phi$ values, and identify the plateaus in both density profiles that correspond to the same gap. The density difference of those two plateaus divided by the difference between $\phi$ values gives the Hall conductance. We use Fig. 2 as an example to show how this procedure works. The plateaus are marked by the same symbol as their corresponding gaps in Fig. 1. Two plateaus marked by the same symbol are the same insulating phase. In Fig. 2(a), for that marked by $\times, \Delta n=0.333-0.25$, and $\Delta n / \Delta \phi=1$, for that marked by $\boldsymbol{\Lambda}, \Delta n=0.667-0.75$, and $\Delta n / \Delta \phi=-1$. In Fig. 2(b), one can get the same quantization number for the plateaus marked by $\times$ and $\boldsymbol{\Lambda}$. In addition, there are more plateaus, such as those marked by $\bigcirc$ and $\mathbf{\square}$ corresponding to the second largest gaps. For that marked by $\bigcirc, \Delta n=$ $0.284-0.201$ and $\Delta n / \Delta \phi=2$, and for that marked by

$\Delta n=0.716-0.802$ and $\Delta n / \Delta \phi=-2$.

While the above examples concentrated on the largest, Landau level type, gaps of the spectrum, the method can be applied to any gap, including the nontrivial smaller gaps in the right-left or central chains. For example, considering two density profiles near $\phi=0.45$ we can observe the Hall conductance of the largest gap in the right chain to be $\Delta n / \Delta \phi=2$. Generally smaller gaps have higher values of Hall conductance; however, experimentally it becomes increasingly harder to observe these gaps, as the corresponding plateaus will become discernible at lower temperatures and higher particle numbers.

In summary, we discussed the experimental conditions for simulating Hofstadter model in rotating optical lattices, such as lattice depth, rotational frequency, and temperature. We calculated the density profile in the presence of a smooth residual trapping potential, and showed how the density plateaus reflect the insulating behavior in a "magnetic field" with incommensurate filling number. We also propose a method to measure the Hall conductance from real space density profiles, without doing transport experiments.

R. O.U. is supported by TÜBITAK. H.Z. and M.Ö. O. would like to thank the Institut Henri Poincaré's program on "Quantum Gases" for hospitality. M. Ö. O. is supported by TÜBITAK-KARIYER Grant No. $104 T 165$ and a TÜBA-GEBIP grant.

*oktel@fen.bilkent.edu.tr

[1] H. Ott et al., Phys. Rev. Lett. 92, 160601 (2004).

[2] M.W. Zwierlein, A. Schirotzek, C.H. Schunck, and W. Ketterle, Science 311, 492 (2006).

[3] G. B. Partridge, W. Li, R. I. Kamar, Y. Liao, and R. G. Hulet, Science 311, 503 (2006).

[4] D. R. Hofstadter, Phys. Rev. B 14, 2239 (1976).

[5] D. J. Thouless, M. Kohmoto, M. P. Nightingale, and M. den Nijs, Phys. Rev. Lett. 49, 405 (1982).

[6] I. Dana, Y. Avron, and J. Zak, J. Phys. C 18, L679 (1985).

[7] J. Kellendonk, Commun. Math. Phys. 258, 751 (2005).

[8] P. Středa, T. Jonckheere, and T. Martin, arXiv:0710.5786v1.

[9] The strongest continuous magnetic field available nowadays is only 45 T. See H. J. Schneider-Muntau, J. Toth, and H.W. Weijers, IEEE Trans. Appl. Supercond. 14, 1245 (2004).

[10] C. Albrecht et al., Phys. Rev. Lett. 86, 147 (2001).

[11] N. R. Cooper et al., Phys. Rev. Lett. 87, 120405 (2001); N. Regnault et al., ibid. 91, 030402 (2003); M. A. Baranov et al., ibid. 94, 070404 (2005).

[12] For bosons in rotating optical lattices, see C. Wu et al., Phys. Rev. A 69, 043609 (2004); M. Polini et al., Laser Phys. 14, 603 (2004); J. W. Reijnders et al., Phys. Rev. Lett. 93, 060401 (2004); H. Pu et al., ibid. 94, 190401 (2005); A. S. Sørensen et al., ibid. 94, 086803 (2005); R. Bhat et al., ibid. 96, 060405 (2006); R. N. Palmer et al., ibid. 96, 180407 (2006); R. O. Umucalılar et al., Phys. Rev. A 76, 055601 (2007).

[13] See Chap. 16.2 of L. Pitaevskii and S. Stringari, BoseEinstein Condensation (Clarendon Press, Oxford, 2003); H. Zhai and T. L. Ho, Phys. Rev. Lett. 99, 100402 (2007); T. L. Ho and Q. Zhou, ibid. 99, 120404 (2007).

[14] S. Tung, V. Schweikhard, and E. A. Cornell, Phys. Rev. Lett. 97, 240402 (2006).

[15] M. Greiner, O. Mandel, T. Esslinger, T. W. Hänsch, and I. Bloch, Nature (London) 415, 39 (2002).

[16] The density profile of fermions in a rotating trap was examined in T.L. Ho and C.V. Ciobanu, Phys. Rev. Lett. 85, 4648 (2000).

[17] P. Středa, J. Phys. C 15, L717 (1982).

[18] M. Köhl, H. Moritz, T. Stöferle, K. Günter, and T. Esslinger, Phys. Rev. Lett. 94, 080403 (2005). 